\title{
Preditores do desconhecimento do status sorológico de HIV entre puérperas submetidas ao teste rápido anti-HIV na internação para o parto
}

\author{
Predictors of unawareness of HIV serostatus among women \\ submitted to the rapid HIV test at admittance for delivery
}

Marcelo de Lima Soares ${ }^{1}$

Maria Inês Couto de Oliveira ${ }^{2}$

Vânia Matos Fonseca ${ }^{3}$

Alexandre dos Santos Brito ${ }^{4}$

Kátia Silveira da Silva ${ }^{3}$

${ }^{1}$ Policlínica Naval Nossa Senhora da Glória. R. Conde de Bonfim 54, Tijuca. 20520-053 Rio de Janeiro RJ. marcelosoares2@yahoo.com.br ${ }^{2}$ Departamento de

Epidemiologia e

Bioestatística, Instituto de

Saúde da Comunidade,

Universidade Federal

Fluminense.

${ }^{3}$ Unidade de Pesquisa Clínica, Instituto Fernandes

Figueira, Fundação Oswaldo Cruz.

${ }^{4}$ Instituto de Estudos em

Saúde Coletiva, Universidade

Federal do Rio de Janeiro.

\begin{abstract}
This article aims to analyze factors associated with unawareness of prenatal HIV serostatus at admittance for delivery. A cross-sectional study was performed in 2006 in "Friends of Children" Hospitals from the High-risk Pregnancy System, belonging to the Unified Health System, in Rio de Janeiro City. Data were collected through interviews applied to 873 rooming-in mothers submitted to a rapid HIV test at the hospital. Prevalence ratios $(P R)$ of the lack of HIV serologic status were estimated by Poisson regression with robust variance, controlled by maternal and familiar socio-demographic characteristics, pregnancy and prenatal care. Prevalence of unawareness of HIV status was $32.2 \%$. Mothers with low educational level, low-income, more than one relationship in the last year, enrolling late in prenatal care, and low number of prenatal visits were more likely to have unknown HIV status. The main predictor for unawareness of HIV serostatus at hospital admittance was the low number of prenatal visits. It is recommended that coverage of $H I V$ testing during prenatal care be broadened with timely delivery of results, improving early access of pregnant women and increasing the number of prenatal visits, focusing on clients with low socio-economic level.
\end{abstract}

Key words Prenatal care, HIV, AIDS serodiagnosis, Cross-sectional studies, Unified Health System
Resumo O objetivo deste artigo é analisar fatores associados ao desconhecimento do status sorológico de HIV do pré-natal na internação ao parto. Estudo transversal realizado em 2006 em Hospitais Amigos da Criança do Sistema de Gestação de Alto Risco do SUS no município do Rio de Janeiro. Foram entrevistadas no alojamento conjunto 873 mães submetidas ao teste rápido anti-HIV no hospital. Razões de prevalência (RP) do desconhecimento do status de HIV foram obtidas por regressão de Poisson com variância robusta, com ajuste por características sociodemográficas maternas e familiares, da gravidez e de assistência pré-natal. A prevalência de status ignorado de HIV foi 32,2\%. Baixa escolaridade materna, baixa renda materna, ter tido mais de um relacionamento no último ano, início tardio do pré-natal e baixo número de consultas pré-natais mostraram-se associados ao desfecho. O mais importante preditor do desconhecimento do status de HIV na internação para o parto foi o baixo número de consultas pré-natais. Recomenda-se a expansão da cobertura da testagem anti-HIV no pré-natal e a entrega de resultados em tempo hábil, com captação precoce da gestante e incremento no número de consultas, enfocando a clientela de baixo nível socioeconômico.

Palavras-chave Assistência pré-natal, HIV, Sorodiagnóstico da AIDS, Estudos transversais, Sistema Único de Saúde 


\section{Introdução}

Uma atenção pré-natal e puerperal qualificada e humanizada ocorre por meio da incorporação de condutas acolhedoras e sem intervenções desnecessárias e do fácil acesso a serviços de saúde de qualidade, com ações que integrem todos os níveis da atenção: promoção, prevenção e assistência à saúde da gestante e do recém-nascido, desde $\mathrm{o}$ atendimento ambulatorial básico ao atendimento hospitalar para alto risco. O Ministério da Saúde preconiza a realização de, no mínimo, 6 consultas de pré-natal, com a realização de exames laboratoriais como $\mathrm{ABO} / \mathrm{Rh}$, Hemoglobina/Hematócrito, Glicemia de jejum, Urina tipo 1, Sorologia para toxoplasmose, Hepatite B, VDRL e anti-HIV ${ }^{1}$.

A AIDS (síndrome da imunodeficiência adquirida) caracteriza-se por uma disfunção grave do sistema imunológico e é causada por um retrovírus denominado vírus da imunodeficiência humana (HIV) $)^{2}$. A testagem anti-HIV no prénatal visa principalmente a prevenção da transmissão vertical do vírus. Esta é a principal via de infecção pelo HIV em crianças e ocorre através da passagem do vírus da mãe para o bebê durante a gestação, o trabalho de parto, o parto propriamente dito, ou através da amamentação. Cerca de 35\% dessa transmissão ocorre durante a gestação, $65 \%$ no peri-parto ${ }^{3,4}$ e há um risco acrescido de transmissão através da amamentação entre $7 \%$ e $22 \%{ }^{5}$.

O exame anti-HIV no pré-natal deve ser realizado através do consentimento da gestante após aconselhamento pré-teste, sendo o seu resultado entregue após aconselhamento pós-teste ${ }^{1}$. Atualmente, este exame é preconizado na primeira consulta de pré-natal, com repetição no início do terceiro trimestre, sempre que possível ${ }^{6}$. Em 2006, na época desta pesquisa, era preconizada a realização de exame anti-HIV no primeiro trimestre da gravidez, e sua repetição ficava dependente da gestante se enquadrar em critérios de vulnerabilidade. Assim, poderia ser realizado novo exame no final da gestação, ou no momento da internação para o parto, através do teste rápido anti-HIV ${ }^{1}$.

No cenário brasileiro vem sendo verificado um aumento gradual na cobertura efetiva do exame anti-HIV na gestação, passando de 52\% no ano de $2002^{7}$ para $62 \%$ em $2006^{8}$. Estudos regionais indicam disparidades na prevalência desta testagem, tendo sido observada uma cobertura de 7,6\% em municípios da Região Norte-Nordeste em $2002^{9}$ e de 90,2\% em Porto Alegre em 2000-2001 ${ }^{10}$.
Estudos que investigaram os fatores de risco para a não realização do exame anti-HIV durante o pré-natal no Brasil indicaram os fatores: adolescência, baixa escolaridade, desconhecimento prévio sobre a prevenção da transmissão vertical, cor de pele não branca, ser solteira, possuir vida sexual ativa durante a gravidez, realização de pré-natal na rede privada, baixo número de consultas pré-natais e realização da última consulta de pré-natal no $2^{\circ}$ trimestre ${ }^{10-12}$.

Já para o não recebimento do resultado do exame anti-HIV no pré-natal, os fatores de risco encontrados em estudos brasileiros foram: possuir idade inferior a 35 anos, cor de pele não branca, baixa escolaridade, residir fora da região metropolitana ou em município pequeno, residir fora da Região Sul brasileira, ter realizado o exame no terceiro trimestre e ausência de aconselhamento ${ }^{7,8,13}$.

Diante da cobertura ainda insuficiente de testagem anti-HIV no pré-natal ${ }^{8}$, a investigação dos fatores associados ao desconhecimento do status sorológico de HIV na internação para o parto torna-se imprescindível. Assim, a compreensão acerca dos entraves ao conhecimento do diagnóstico de HIV do pré-natal poderá contribuir para uma melhor intervenção do governo, através de seus programas, para a prevenção da transmissão vertical do vírus, gerando maior ganho para a saúde e o bem-estar do binômio mãe-filho.

O objetivo deste estudo foi analisar os fatores associados ao desconhecimento do status sorológico de HIV entre puérperas internadas em alojamento conjunto submetidas ao teste rápido anti-HIV em Hospitais Amigos da Criança ${ }^{14}$ no município do Rio de Janeiro.

\section{Métodos}

Trata-se de estudo transversal, que faz parte do projeto de pesquisa "Gênero, Poder e Cidadania: a mulher é sujeito no processo decisório da amamentação ao nascimento quando o status de HIV é ignorado pelo serviço?", aprovado pelos Comitês de Ética da Secretaria Municipal de Saúde do Rio de Janeiro e do Instituto Fernandes Figueira/ Fiocruz. A população alvo da pesquisa foi composta por todas as mulheres que realizaram o teste rápido anti-HIV por ocasião do parto, internadas em regime de alojamento conjunto, no período de 11 de setembro a 11 de dezembro de 2006 nos cinco Hospitais Amigos da Criança pertencentes ao Sistema de Gestação de Alto Risco do Município do Rio de Janeiro ${ }^{15}$. Estes eram hospitais próprios do Sistema Único de Saúde. 
Os dados foram obtidos através de entrevista às mães no puerpério imediato mediante assinatura de termo de consentimento livre e esclarecido.

Como o presente estudo visou investigar os fatores associados ao status sorológico ignorado de HIV do pré-natal, da pesquisa original ${ }^{15}$ foram excluídas as 82 mães que não haviam realizado qualquer consulta de pré-natal (8,6\% das 955 mães entrevistadas), restando 873 mães para a análise.

A entrada de dados foi realizada por meio do programa Epi-Info ${ }^{\circledR}$ e a análise por meio do programa SPSS16 ${ }^{\circledR}$.

O desconhecimento ( $\operatorname{sim} v s$. não) do status sorológico de HIV do pré-natal foi considerado a variável desfecho neste estudo. A Figura 1 elucida as variáveis pesquisadas, dentro de um modelo teórico hierarquizado de determinação ${ }^{16}$ desse desfecho. Entre as variáveis de exposição, foram consideradas distais as sociodemográficas e as reprodutivas maternas: idade, cor da pele, escolaridade, trabalho, renda, presença de companheiro, número de relacionamentos no último ano, paridade, e as variáveis familiares: número de bens e renda per capita. As variáveis de exposição consideradas proximais foram características da gestação e de assistência pré-natal: gravidez planejada, trimestre de início do prénatal e número de consultas pré-natais.
Primeiramente foi desenvolvida uma análise univariada para conhecimento do perfil das mães. Em seguida foi realizada uma análise bivariada entre cada variável de exposição, expressa de forma dicotômica, e o desfecho, o desconhecimento do status sorológico de HIV do pré-natal. Foram também realizados testes de hipóteses de qui-quadrado e obtidas razões de prevalência (RP) brutas com seus respectivos intervalos de confiança de 95\% (IC 95\%). As variáveis de exposição que, na análise bivariada, mostraram-se associadas com o desfecho com nível de significância de até $20 \%$ no teste de qui-quadrado ( $\mathrm{p}$-valor $<0,20$ ) foram selecionadas para a análise multivariada.

Razões de prevalência ajustadas foram obtidas por modelo de regressão de Poisson com variância robusta, pois o desfecho apresentou uma prevalência elevada ${ }^{17}$. A inclusão das variáveis no modelo seguiu uma abordagem hierarquizada $^{16}$, sendo incluídas primeiramente as distais. Aquelas que atingiram um nível de significância de até $5 \%(\mathrm{p}$-valor $<0,05)$ permaneceram no modelo. Posteriormente, foram adicionadas as variáveis proximais, permanecendo no modelo final aquelas com um nível de significância de até $5 \%$ (p-valor < 0,05), assim como as distais selecionadas anteriormente.

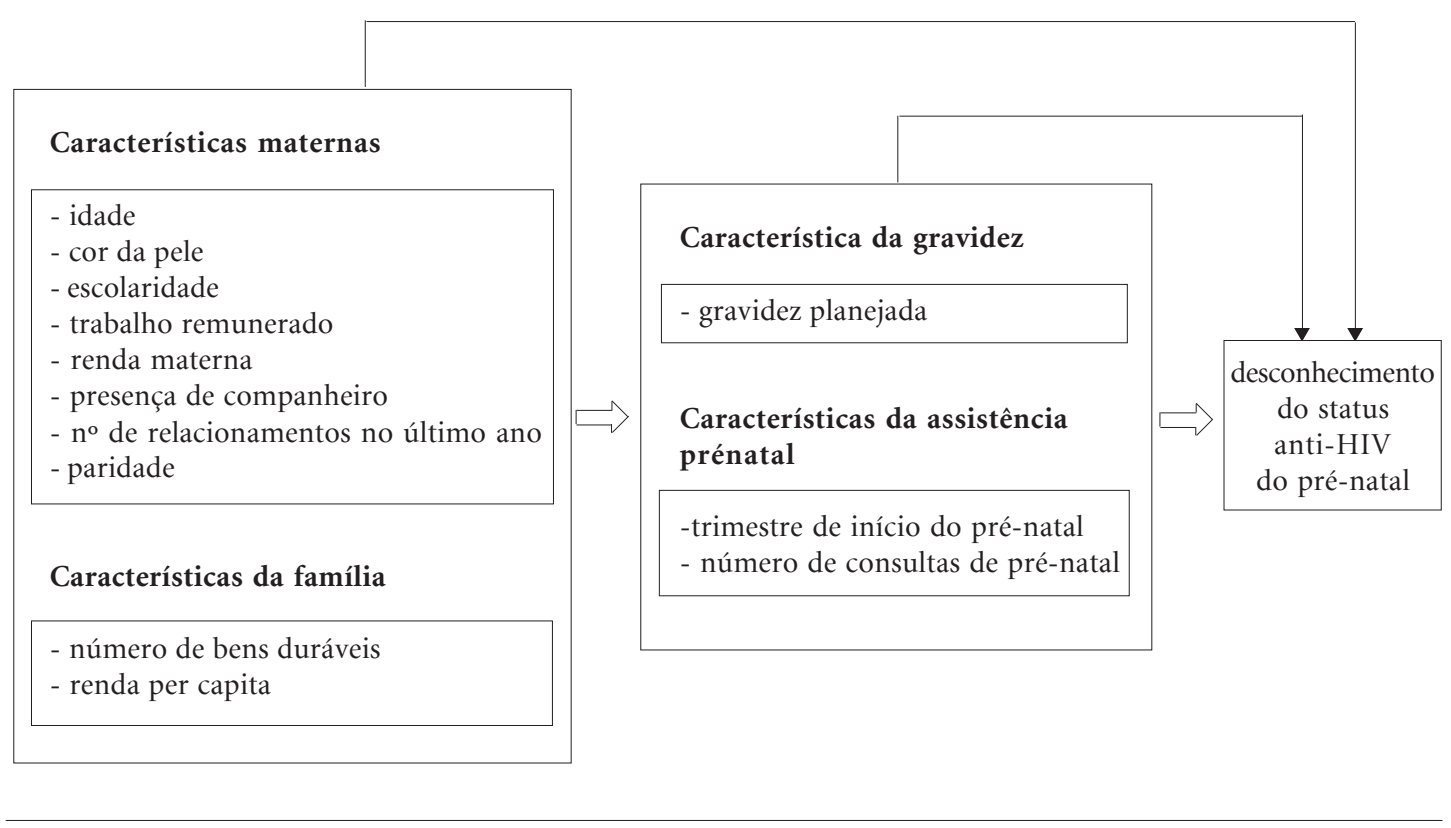

Figura 1. Modelo teórico de determinação do desconhecimento do status sorológico de HIV do pré-natal, Município do Rio de Janeiro, 2006. 


\section{Resultados}

Da população do estudo formada por $873 \mathrm{mu}$ lheres, apenas $592(67,8 \%)$ receberam o resultado de algum exame anti-HIV no pré-natal. Tinham status ignorado de HIV 281 mães (32,2\%), por não terem realizado o exame (129 mães) ou por não terem recebido o resultado deste exame, apesar de o haverem realizado (152 mães).

Quanto às características das 873 mães, 27,0\% eram adolescentes, $72,2 \%$ declararam-se de cor não branca e $41,1 \%$ não haviam completado o ensino fundamental. A maioria não exercia trabalho remunerado $(52,0 \%)$ e $62,9 \%$ tinham uma renda menor que um salário mínimo vigente na época ( $\mathrm{R} \$ 350,00)$. Tinham companheiro $88,8 \%$ das mães, $8,8 \%$ tinham tido outro relacionamento no último ano, além do pai do bebê, e $41,7 \%$ eram primíparas. Dispunham de 0 a 3 bens no domicílio $37,0 \%$ das mães, tendo $83,4 \%$ das mães uma renda familiar per capita inferior ou igual a um salário mínimo. A maior parte das mães não teve uma gravidez planejada $(66,9 \%)$ e iniciou o pré-natal no primeiro ou segundo trimestre da gravidez $(87,8 \%)$. Realizaram 6 ou mais consultas de pré-natal $66,8 \%$ das mães.

$\mathrm{Na}$ análise bivariada as variáveis distais cor da pele, escolaridade, trabalho, renda materna, presença de companheiro, número de relacionamentos no último ano, paridade, número de bens e renda per capita mostraram-se associados ao desfecho com nível de significância de 20\% (Tabela 1), bem como as variáveis proximais: gravidez planejada, número de consultas de pré-natal e trimestre de início de pré-natal (Tabela 2).

$\mathrm{Na}$ análise multivariada mostraram-se associados positivamente ao desconhecimento do status sorológico de HIV do pré-natal a baixa esco-

Tabela 1. Prevalência e Razão de Prevalência (RP) bruta do desconhecimento do status sorológico de HIV do pré-natal segundo características maternas e familiares, Município do Rio de Janeiro, 2006.

\begin{tabular}{|c|c|c|c|c|c|c|}
\hline \multirow[b]{2}{*}{ Variáveis } & \multicolumn{2}{|c|}{ Frequência } & \multirow{2}{*}{$\begin{array}{c}\text { Prevalência } \\
\%\end{array}$} & \multirow[b]{2}{*}{ RP bruta } & \multirow[b]{2}{*}{ IC $95 \%$} & \multirow[b]{2}{*}{ p-valor } \\
\hline & $\mathrm{N}$ & $\%$ & & & & \\
\hline \multicolumn{7}{|l|}{ Idade } \\
\hline Adulta & 637 & 73,0 & 31,4 & 1 & & \\
\hline Adolescente & 236 & 27,0 & 34,3 & 1,093 & $0,88-1,35$ & 0,411 \\
\hline \multicolumn{7}{|l|}{ Cor da pele } \\
\hline Branca & 243 & 27,8 & 28,0 & 1 & & \\
\hline Não branca & 630 & 72,2 & 33,8 & 1,21 & $0,96-1,52$ & 0,099 \\
\hline \multicolumn{7}{|l|}{ Escolaridade } \\
\hline Ensino Médio incompleto & 359 & 41,1 & 42,1 & 1,66 & $1,37-2,95$ & 0,000 \\
\hline$>$ Ensino Médio completo & 514 & 58,9 & 25,3 & 1 & & \\
\hline \multicolumn{7}{|l|}{ Trabalho materno } \\
\hline Não & 454 & 52,0 & 35,7 & 1 & & \\
\hline Sim & 419 & 48,0 & 28,4 & 0,80 & $0,65-0,97$ & 0,021 \\
\hline \multicolumn{7}{|l|}{ Renda materna } \\
\hline > 1 salário mínimo & 324 & 37,1 & 22,8 & 1 & & \\
\hline < 1 salário mínimo & 549 & 62,9 & 37,7 & 1,65 & $1,31-2,07$ & 0,000 \\
\hline \multicolumn{7}{|l|}{ Companheiro } \\
\hline Tem companheiro & 775 & 88,8 & 31,2 & 1 & & \\
\hline Não tem companheiro & 98 & 11,2 & 39,8 & 1,27 & $0,98-1,66$ & 0,087 \\
\hline \multicolumn{7}{|c|}{ No de relacionamentos no último ano } \\
\hline 1 & 796 & 91,2 & 30,5 & 1 & & \\
\hline$>2$ & 77 & 8,8 & 49,4 & 1,62 & $1,26-2,07$ & 0,000 \\
\hline \multicolumn{7}{|l|}{ Paridade } \\
\hline Primípara & 362 & 41,7 & 27,3 & 1 & & \\
\hline Multípara & 506 & 58,3 & 35,0 & 1,28 & $1,04-1,57$ & 0,017 \\
\hline \multicolumn{7}{|l|}{ Número de bens } \\
\hline 4 a 5 & 548 & 63,0 & 27,6 & 1 & & \\
\hline Até 3 & 322 & 37,0 & 40,1 & 1,45 & $1,20-1,76$ & 0,000 \\
\hline \multicolumn{7}{|l|}{ Renda per capita } \\
\hline > 1 salário mínimo & 134 & 16,6 & 17,2 & 1 & & \\
\hline$\leq 1$ salário mínimo & 675 & 83,4 & 35,0 & 2,04 & $1,39-3,00$ & 0,000 \\
\hline
\end{tabular}


laridade e a baixa renda maternas, a mãe ter tido mais de um relacionamento no último ano, o início tardio do pré-natal e o baixo número de consultas pré-natais (Tabela 3).

\section{Discussão}

Apesar do exame anti-HIV estar disponível nos serviços pré-natais do município do Rio de Janeiro desde $1996^{18}$, neste estudo, realizado dez anos depois, apenas $2 / 3$ das mulheres estudadas fizeram o exame e receberam o seu resultado durante a gestação. Este cenário foi bem menos favorável do que o encontrado entre 2000 e 2001 em Porto Alegre ${ }^{19}$, onde praticamente $90 \%$ das puérperas haviam sido testadas no pré-natal. Em pesquisa nacional realizada em 2006 apenas 62,3\% das parturientes brasileiras dispunham do resultado do exame anti-HIV da gestação na internação para o parto, sendo esta proporção de 85,8\% na região Sul e de $73,7 \%$ na região Sudeste, tendo os piores resultados sido encontrados nas regiões Norte $(45,9 \%)$ e Nordeste $(40,6 \%)^{8}$. Em 2006, o Ministério da Saúde já preconizava que pelo menos um exame anti-HIV fosse realizado neste período, e que seu resultado fosse disponibilizado às gestantes mediante aconselhamento ${ }^{1}$. Os

Tabela 2. Prevalência e Razão de Prevalência (RP) bruta do desconhecimento do status sorológico de HIV do pré-natal segundo características da gravidez e da assistência pré-natal (PN), Município do Rio de Janeiro, 2006.

\begin{tabular}{|c|c|c|c|c|c|c|}
\hline \multirow[b]{2}{*}{ Variáveis } & \multicolumn{2}{|c|}{ Frequência } & \multirow{2}{*}{$\begin{array}{c}\text { Prevalência } \\
\%\end{array}$} & \multirow[b]{2}{*}{ RP bruta } & \multirow[b]{2}{*}{ IC $95 \%$} & \multirow[b]{2}{*}{ p-valor } \\
\hline & $\mathbf{N}$ & $\%$ & & & & \\
\hline \multicolumn{7}{|l|}{ Gravidez planejada } \\
\hline Sim & 289 & 33,1 & 26,6 & 1 & & \\
\hline Não & 584 & 66,9 & 34,9 & 1,31 & $1,05-1,64$ & 0,014 \\
\hline \multicolumn{7}{|l|}{ Trimestre início do PN } \\
\hline $1^{\circ}$ e $2^{\circ}$ trimestre & 763 & 87,8 & 28,0 & 1 & & \\
\hline $3^{\circ}$ trimestre & 106 & 12,2 & 62,3 & 2,22 & $1,84-2,68$ & 0,000 \\
\hline \multicolumn{7}{|l|}{ No de consultas de PN } \\
\hline 6 a 23 & 577 & 66,8 & 23,4 & 1 & & \\
\hline 1 a 5 & 287 & 33,2 & 49,1 & 2,10 & $1,74-2,54$ & 0,000 \\
\hline
\end{tabular}

Tabela 3. Razão de Prevalência (RP) bruta e ajustada do desconhecimento do status sorológico de HIV do pré-natal segundo características maternas, da família e de assistência pré-natal (PN), Município do Rio de Janeiro, 2006.

\begin{tabular}{|c|c|c|c|c|}
\hline Variáveis & RP bruta & RP ajustada & $\mathrm{IC}_{95 \%}$ & p-valor \\
\hline \multicolumn{5}{|l|}{ Escolaridade materna } \\
\hline$>$ Ensino Médio completo & 1 & 1 & & \\
\hline Ensino Médio incompleto & 1,66 & 1,38 & $1,13-1,67$ & 0,001 \\
\hline \multicolumn{5}{|l|}{ Renda materna } \\
\hline > 1 salário mínimo & 1 & 1 & & \\
\hline$<1$ salário mínimo & 1,65 & 1,45 & $1,16-1,82$ & 0,001 \\
\hline \multicolumn{5}{|c|}{ No de relacionamentos no último ano } \\
\hline 1 & 1 & 1 & & \\
\hline 2 ou mais & 1,62 & 1,31 & $1,02-1,68$ & 0,035 \\
\hline \multicolumn{5}{|l|}{ Trimestre do início do PN } \\
\hline $1^{\circ}$ ou $2^{\circ}$ trimestre & 1 & 1 & & \\
\hline $3^{\circ}$ trimestre & 2,22 & 1,49 & $1,19-1,87$ & 0,001 \\
\hline \multicolumn{5}{|l|}{ Número de consultas de PN } \\
\hline 6 a 23 & 1 & 1 & & \\
\hline 1 a 5 & 2,10 & 1,66 & $1,33-2,07$ & 0,000 \\
\hline
\end{tabular}

$\mathrm{n}=858$ 
níveis encontrados, ainda insatisfatórios, de testagem para o diagnóstico do HIV no pré-natal, refletem oportunidades perdidas de prevenção da transmissão vertical, pois o diagnóstico precoce de HIV com o uso de drogas antirretrovirais na gestação, no trabalho de parto e pelo concepto podem reduzir a transmissão vertical a menos de $2 \%{ }^{20}$.

No cenário internacional foram encontrados entraves à testagem anti-HIV no pré-natal de diferentes ordens. Nos Estados Unidos não dispor de plano de saúde ${ }^{21}$ ou ter lacunas no seguro saúde ${ }^{22}$ se mostraram barreiras importantes. Em Uganda foram identificados como entraves a indisponibilidade de serviços de testagem anti-HIV e a recusa à realização do exame, por medo ou pela ausência de benefícios percebidos pela mulher caso houvesse detecção de soropositividade, como a falta de tratamento gratuito e de leite artificial para seu filho ${ }^{23}$. Já no Sistema Único de Saúde brasileiro tanto o exame anti-HIV quanto o tratamento são gratuitos, e apesar deste exame ser de caráter voluntário ${ }^{1}$, são raros os casos de recusa entre gestantes ${ }^{10,12}$.

Para a presente investigação foram analisadas apenas as mães que tinham realizado ao menos uma consulta de pré-natal, para as quais poderia ter sido oferecido e realizado o exame anti-HIV com resultado disponível oportunamente na gestação. Caso as mães que não realizaram pré-natal tivessem sido incluídas na análise, o cenário encontrado seria ainda mais grave, passando de $32 \%$ para $38 \%$ o desconhecimento do status sorológico do HIV na gestação entre as mulheres submetidas ao teste rápido anti-HIV.

Cabe se considerar algumas limitações do presente estudo, como um potencial viés de memória quanto à realização do exame anti-HIV na gestação e ao período de sua realização, uma vez que estes dados foram obtidos por meio de entrevista às mães no puerpério imediato. Uma limitação à validade externa dos nossos resultados deve-se ao fato da população de estudo ter se restringido às puérperas submetidas ao teste rápido anti-HIV por ocasião do parto, que corresponderam a $28,5 \%$ da clientela internada em alojamento conjunto no período da pesquisa ${ }^{15}$. Provavelmente estas mulheres apresentavam um perfil de assistência pré-natal mais desfavorável, tendo por isso sido selecionadas para a realização do teste rápido anti-HIV. Outro aspecto a ser considerado é que em estudos transversais, nem sempre a relação temporal entre exposição e desfecho fica bem estabelecida. No entanto, na presente pesquisa as variáveis de exposição estu- dadas foram características maternas, familiares, da gestação e da assistência pré-natal, que precederam o desconhecimento do status de HIV na internação para o parto, sendo possível, portanto, considerá-las fatores de risco para o desfecho estudado.

Neste estudo, o mais importante preditor do desconhecimento do status de HIV da gestação foi o baixo número de consultas pré-natais, que gerou um aumento da prevalência do desfecho em $66 \%$. Este resultado foi consistente ao encontrado no Zimbabwe ${ }^{24}$ e também em Porto Ale$\mathrm{gre}^{10}$, onde as mães que realizaram menos de seis consultas pré-natais apresentaram uma prevalência do desfecho 2,7 vezes maior. Veloso et al. ${ }^{12}$, em estudo realizado em 12 cidades brasileiras, observaram que a realização de apenas uma ou duas consultas pré-natais dobrou a prevalência do desfecho. O segundo fator mais importante para o desconhecimento do status sorológico de HIV na gestação foi o início do pré-natal no terceiro trimestre, que aumentou a prevalência do desfecho em $49 \%$. Ramos et al. ${ }^{13}$, em Recife, encontraram um resultado ainda mais desfavorável, o início do pré-natal no terceiro trimestre de gestação aumentando a chance do desconhecimento do status sorológico de HIV do pré-natal em 11,6 vezes. Quanto menos e mais tardiamente a gestante frequenta a assistência pré-natal, menos oportunidade tem de realizar os exames preconizados para a gestação e de receber seus resultados em tempo hábil.

Em nosso estudo não só a precária assistência pré-natal, mas também a vida sexual e o baixo nível socioeconômico contribuíram para o desfecho. A mulher ter tido outro(s) relacionamento(s) no último ano, além do pai do bebê, aumentou a prevalência do desfecho em $31 \%$, de forma semelhante à identificada em pesquisa nacional ${ }^{11}$, onde as mulheres com vida sexual ativa na gravidez, apesar de mais vulneráveis, eram menos testadas para o HIV.

O ganho materno inferior a um salário mínimo aumentou a prevalência do desconhecimento do status sorológico de HIV do pré-natal em $44 \%$. Nesta mesma pesquisa nacional ${ }^{11}$, conduzida para investigar oportunidades perdidas para a prevenção da sífilis congênita e da transmissão vertical do HIV foi encontrado um efeito dose resposta: quanto mais baixa a renda familiar, maior a chance da mulher não possuir sorologia para estes dois exames. A avaliação do programa de controle da transmissão vertical do HIV em maternidades do Projeto Nascer verificou que as parturientes com uma renda média per capita superior a $R \$ 400,00$ 
apresentaram uma chance três vezes maior de terem sido testadas durante o pré-natal ${ }^{25}$. Também a baixa escolaridade das mulheres pesquisadas esteve associada a uma maior prevalência do desfecho, conforme descrito em estudos realizados no Brasil ${ }^{7,8,11-13,25}$ e na África ${ }^{24}$.

Um dos princípios do Sistema Único de Saúde é a equidade, ou seja, a prioridade na oferta de ações e serviços aos segmentos populacionais que enfrentam maiores riscos de adoecer ou morrer em decorrência da distribuição desigual de ren$\mathrm{da}$, bens e serviços ${ }^{26}$. Portanto, a clientela que deveria ser cuidada com mais atenção é justamente a que vem tendo menos acesso à sorologia de HIV na gestação.
Há, portanto, a necessidade do aprimoramento de políticas públicas para a redução das desigualdades sociais e de políticas de saúde voltadas para a captação precoce das gestantes para o pré-natal e de assistência humanizada com vistas à fixação desta clientela no pré-natal. Recomenda-se, também, que seja estendida a cobertura da testagem anti-HIV no pré-natal, sendo agilizado o processo de entrega dos resultados, para que a parturiente chegue ao hospital com status de HIV conhecido. Preferencialmente, a realização do exame anti-HIV deve ser solicitada na primeira consulta de pré-natal e este status sorológico deve ser confirmado no início do último trimestre de gestação ${ }^{6}$.

\section{Colaboradores}

ML Soares, MIC Oliveira, VM Fonseca, AS Brito e KS Silva participaram igualmente de todas as etapas de elaboração do artigo. 


\section{Referências}

1. Brasil. Ministério da Saúde (MS). Pré-Natal e Puerpério: Atenção Qualificada e Humanizada. Manual Técnico. Brasília: MS; 2005.

2. Barré-Sinoussi F, Cherman JC, Rey F, Nugeyre MT, Chamaret S, Gruest J, Dauguet C, Axler-Blin C, Vézinet-Brun F, Rouzioux C, Rozenbaum W, Montagnier L. Isolation of a T-lymphtropic retrovirus from a patient at risk for Acquired Immune Deficiency Syndrome (AIDS). Science 1983; 220(4599):868-871.

3. Bertolli J, St Louis ME, Simonds RJ, Nieburg P, Kamenga M, Brown C, Tarande M, Quinn T, Ou CY. Estimating the timing of mother-to-child transmission of human immunodeficiency virus in a breast-feeding population in Kinshasa, Zaire. J Infect Dis 1996; 174(4):722-726.

4. Rouzioux C, Costagliola D, Burgard M, Blanche S, Griscelli C, Valleron AJ. HIV Infection in Newborns French Collaborative Study Group. Estimated timing of mother-to-child human immunodeficiency virus type 1 (HIV-1) transmission by use of a Marcov model. Am J Epidemiol 1995; 142(12):1330-1337.

5. Dunn TDT, Newll ML, Ades AE, Peckham CS. Risk of human immunodeficiency virus type 1 transmission through breastfeeding. Lancet 1992; 340(8819): 585-588.

6. Brasil. Ministério da Saúde (MS). Secretaria de Vigilância em Saúde. Programa Nacional de DST e AIDS. Protocolo para a prevenção de transmissão vertical de HIV e sifilis - Manual de Bolso. Brasília: MS; 2007.

7. Souza Júnior PRB, Szwarcwald CL, Barbosa Júnior A, de Carvalho MF, Castilho EA. Infecção pelo HIV durante a gestação: Estudo-Sentinela Parturiente, Brasil, 2002. Rev Saude Publica 2004; 38(6):764-772.

8. Szwarcwald CL, Barbosa Júnior A, Souza Júnior PRB, Lemos KRV, Frias PG, Luhm KR, Holcman MM, Esteves MA. HIV testing during pregnancy: use of secondary data to estimate 2006 test coverage and prevalence in Brazil. Braz J Infect Dis 2008; 12(3):167-172.

9. Chrestani MAD, Santos IS, Cesar JA, Winckler LS, Gonçalves TS. Assistência à gestação e ao parto: resultados de dois estudos transversais em áreas pobres das regiões Norte e Nordeste do Brasil. Cad Saude Publica 2008; 24(7):1609-1618.

10. Rosa H, Goldani MZ, Scanlon T, Silva AAM, Giugliani EJ, Agranonik M, Tomkins A. Barriers for HIV testing during pregnancy in Southern Brazil. Rev Saude Publica 2006; 40(2):220-225.

11. Rodrigues CS, Guimarães MDC, César CC. Missed opportunities for congenital syphilis and HIV perinatal transmission prevention. Rev Saude Publica 2008; 42(5):851-858.

12. Veloso VG, Portela MC, Vasconcellos MTL, Matzenbacher ALR, Grinsztejn B, Bastos FI. HIV testing among pregnant women in Brazil: rates and predictors. Rev Saude Publica 2008; 42(5):859-867.

13. Ramos VOX, Lacerda HR, Ximenes RAA. Unawareness of HIV status in pregnancy, delay in testing and conflict between information on antenatal card and interview in Recife, Brazil. Int J STD \& AIDS 2009; 20(7):493-498.

14. Saadeh R, Akré J. Ten steps to successful breastfeeding: a summary of the rationale and scientific evidence. Birth 1996; 23(3):154-160.
15. Oliveira MIC, da Silva KS, Gomes Junior SC, Fonseca VM. Resultado do teste rápido anti-HIV após o parto: uma ameaça à amamentação ao nascimento. Rev Saude Publica 2010; 44(1):60-69.

16. Victora CG, Huttly SR, Fuchs SC, Olinto MTA. The role of conceptual frameworks in epidemiological analysis: a hierarchical approach. Int J Epidemiol 1997; 26(1):224-227.

17. Coutinho LMS, Scazufca M, Menezes PR. Métodos para estimar razão de prevalência em estudos de corte transversal. Rev Saude Publica 2008; 42(6):992998.

18. Rio de Janeiro. Secretaria Municipal de Saúde. Transmissão Vertical do HIV. Município do Rio de Janeiro-2005. Rio de Janeiro: Secretaria Municipal de Saúde; 2006.

19. Goldani MZ, Rosa H, Giugliani E, Tomkins A, Scanlon T, Castilhos K, Feldens L, Tomkins A. Voluntary counselling and HIV testing during antenatal care in Southern Brazil. Rev Saude Publica 2003; 37(5):552-558.

20. Centers for Disease Control and Prevention. Revised recommendations for HIV screening of pregnant women. MMWR Morb Mortal Wkly Rep 2001; 50(RR-19):63-86.

21. Anderson JE, Sansom S. HIV testing in a national sample of pregnant US women: Who is not getting tested? AIDS Care 2007; 19(3):375-380.

22. Lawrence JM, Liu IA, Towner WJ. Trends and correlates of HIV testing during pregnancy in racially/ ethnically diverse insured population, 1997-2006. Matern Child Health J 2009; 13(5):633-640.

23. Karamagi CAS, Tumwine JK, Tylleskar T, Heggenhougen K. Antenatal HIV testing in rural eastern Uganda in 2003: incomplete rollout of the prevention of mother-to-child transmission of HIV programme? BMC Int Health Hum Rights 2006; 6:6.

24. Perez F, Zvandaziva C, Engelsmann B, Dabis F. Acceptability of routine HIV testing ("Opt-Out") in antenatal services in two rural districts of Zimbabwe. J Acquir Immune Defic Syndr 2006; 41(4):514-520.

25. Santos EM, Reis AC, Westman S, Alves RG. Avaliação do grau de implantação do programa de controle da transmissão vertical do HIV em maternidades do "Projeto Nascer". Epidemiol Serv Saúde 2010; 19(3):257-269.

26. Vasconcelos CM, Pasche DF. O Sistema Único de Saúde. In: Campos GWS, Minayo MCS, Akerman M, Drumond Júnior M, Carvalho YM, organizadores. Tratado de Saúde Coletiva. São Paulo, Rio de Janeiro: Hucitec, Ed. Fiocruz; 2006. p. 531-561.

Artigo apresentado em 15/01/2012 Aprovado em 20/02/2012

Versão final apresentada em 19/03/2012 